\title{
Surgery to Remove Adrenocortical Oncocytic Carcinoma from an Asian Male with Scoliosis
}

\author{
Hongtao Liu \\ Dalian Medical University \\ Kai Huang ( $\nabla$ huangkai_2015@163.com ) \\ Yangzhou University Medical college https://orcid.org/0000-0002-3968-0512 \\ Yehua Wang \\ Yangzhou University Medical college \\ Dan Liu \\ Yangzhou University Medical college \\ Xiao Gu \\ Yangzhou University Medical college
}

\section{Case report}

Keywords: adrenocortical carcinoma, oncocytoma, scoliosis, surgery, case report

Posted Date: April 8th, 2021

DOI: https://doi.org/10.21203/rs.3.rs-391042/v1

License: (c) (1) This work is licensed under a Creative Commons Attribution 4.0 International License. Read Full License 


\section{Abstract}

Background: Adrenocortical oncocytoma is a rare type of adrenocortical carcinoma. Its clinical characteristics and biological behaviour need to be further evaluated after the accumulation of cases.

Case presentation: We report a case of adrenocortical oncocytic carcinoma in an Asian male with scoliosis. We performed an operation on this patient. Because the patient's scoliosis was limited during the operation and the tumor protruded into the chest, we decided to adopt open surgery in the supine position. During the operation, we found a tumor about $8 \mathrm{~cm}$ in diameter in the right adrenal region. There was local adhesion with the surrounding tissues. The surface blood vessels of the tumor were distended. After careful dissection and ligation of the blood vessels around the tumor, the adrenal tumor was completely dissociated and removed. The patient recovered well, and his hypertension was controlled after surgery. Pathological results confirmed the diagnosis of adrenocortical oncocytic carcinoma. Pathological features showed that the tumor cells were arranged in nests, with pathological mitosis, abundant cytoplasm, eosinophilia, and invasion of adipose tissue. Immunohistochemistry showed that Ki67 index was more than $15 \%$.

Conclusions: The incidence rate of eosinophilic adrenocortical carcinoma is very low. CT scans and other imaging examination methods are not specific. For larger adrenal tumors, the diagnosis of this disease should be considered. For patients with adrenal cortical carcinoma who have not yet metastasized, we can achieve sound treatment effects and reduce recurrence by removing the tumor, retroperitoneal fat around the tumor and locoregional lymph nodes.

\section{Introduction}

Adrenocortical oncocytic carcinoma is a rare type of adrenocortical carcinoma[1]. Compared with other types of adrenocortical carcinoma, the progression of this kind is relatively slow. Its clinical characteristics and biological behaviour need to be further evaluated. In October 2020, on the basis of sufficient preoperative preparation, we performed surgery on a patient with an adrenal tumor complicated with scoliosis. Postoperative pathology confirmed that the patient had adrenocortical oncocytic carcinoma, and the treatment effect was satisfactory. The report is as follows.

\section{Case Presentation}

A 72-year-old man was admitted to our hospital for treatment due to the discovery of an adrenal tumor on the right side for more than half a month. The patient had no back pain, haematuria or fever. He had hypertension for 6 years but no abnormal palpitation. His scoliosis was caused by spinal trauma 40 years prior (Fig. 1A). His blood pressure was $160 / 96 \mathrm{mmHg}$. A CT scan showed a tumor in the right adrenal area measuring $78 \mathrm{~mm} \times 68 \mathrm{~mm}$ (Fig. 1B). The boundary of the mass was clear with homogeneous enhancement. There was no local invasion or lymph node enlargement. Preoperative examination showed that the urinary vanillylmandelic acid level was $17.5 \mathrm{mg} / 24 \mathrm{hr}$. Serum aldosterone 
and cortisol levels were normal. Before the operation, an a1 receptor blocker was used to control blood pressure for 2 weeks. After his blood pressure and heart rate were stable, an operation was performed.

Because the patient's scoliosis was limited during the operation and the tumor protruded into the chest, we decided to adopt open surgery in the supine position. An incision was made under the costal margin, and the surrounding organs were pushed. The tumor was found in the right adrenal region. There was local adhesion with the surrounding tissues. The surface blood vessels of the tumor were distended. After the blood vessels around the tumor were ligated and removed, the tumor was completely removed (Fig. 1C). The blood loss was approximately $300 \mathrm{ml}$, and the highest intraoperative blood pressure was $182 / 110 \mathrm{mmHg}$.

Postoperative pathological results were as follows (Fig. 2): the tumor cells were arranged in nests with heteromorphic cells; there were obvious nucleoli, pathological mitosis, abundant cytoplasm and eosinophilic cells; and the tumor cells invaded the adipose tissue and had obvious stromal blood vessels. Immunohistochemistry showed the following: vimentin (+), NSE (-), Syn (+), Inhibin (+), Melana (+), S-100 $(-)$, Desmin (-), Ki67 (approximately 15\%+) and Alk (-). No lymph node metastasis was found. The pathological diagnosis was adrenocortical oncocytic carcinoma.

The patient's blood pressure was stable after the operation. After 3 months of follow-up, the patient recovered well without taking antihypertensive drugs. There was no disease recurrence.

\section{Discussion}

Adrenocortical carcinoma is a rare malignant tumor, with an incidence rate of only 1 to 4 persons every 2 million, and includes the oncocytic, mucinous, sarcomatous and other tissue types[2]. Patients with adrenocortical carcinoma have a poor prognosis and are prone to relapse and metastasis. The median overall survival duration is only 3 to 4 years. Patients with adrenocortical oncocytic carcinoma tend to have larger tumors, a lower recurrence rate and a better prognosis than those with other subtypes[3].

Most patients with adrenocortical carcinoma have no obvious symptoms. When the tumor grows gradually, they may have low back pain. Approximately $30 \%$ of adrenocortical oncocytic tumors secrete hormones, which may lead to hypercortisolism and personality abnormalities.

Imaging examinations are of limited value in the diagnosis of adrenocortical oncocytic carcinoma. CT scans and MRI are the commonly used examination methods. Recently, 18F-FDG PET/CT was used in the diagnosis of adrenocortical carcinoma. PET/CT showed that the uptake of FDG in the tumor area was intense but showed inhomogeneous enhancement, while there was no uptake in the adrenal hyperplasia area. This may be related to the overexpression of GLUT1 and hexokinase II in the tumor area[4].

The Weiss scoring system is the most important method to differentiate benign and malignant adrenocortical tumors[5]. The main criteria include mitosis greater than 5/50 HPF, atypical mitosis and venous invasion. The secondary criteria include tumor diameter $>10 \mathrm{~cm}$ and weight $>200 \mathrm{~g}$, tissue necrosis, capsular invasion and blood sinus invasion. If it meets one of the primary criteria, the tumor can 
be diagnosed as malignant. If only one or more secondary criteria are met, it can be considered potentially malignant. If it does not conform to any of the main criteria orsecondary criteria, the tumor can be considered benign. In this report, pathological imaging showed that mitosis was more than 5/50 HPF, and tumor cells invaded the adipose tissue. Therefore, the diagnosis was adrenocortical oncocytic carcinoma. Duregon evaluated 225 patients with adrenocortical carcinoma and found that patients with higher Weiss scores had worse prognoses[2].

A high Ki67 index is associated with tumor growth and tissue differentiation ability. For patients with adrenocortical oncocytic tumors, the Ki67 index is very important for diagnosis and prognosis[6]. Pathologic results of this patient showed a Ki67 index greater than 15\%, which was consistent with the manifestation of adrenocortical carcinoma. The negative immunohistochemical staining of Syn and Inhibin is often related to the poor prognosis of patients with adrenocortical carcinoma[7]. This patient may have a low chance of recurrence because of the positive pathological staining of Syn and Inhibin. It has also been confirmed that patients with adrenocortical oncocytic carcinoma have better prognoses than those with other subtypes[3].

Surgery is still the main method for localized adrenocortical oncocytic carcinoma. Retroperitoneal laparoscopic surgery or open surgery through the 11th intercostal incision in the lumbar back can be used. In this case, due to the large volume of the tumor and scoliosis, we adopted an open operation with a subcostal incision to ensure good visual exposure. During the operation, we removed the tumor and its surrounding retroperitoneal fat as a whole. Meanwhile, we performed local lymph node dissection to ensure the resection scope. Although the patient had scoliosis and a thoracic deformity, we still gently pushed the liver, diaphragm, intestinal tube and other surrounding organs to acquire good surgical vision. This ensured the successful resection of the tumor.

For patients with recurrence risk after resection, mitotane can be used as adjuvant therapy[8]. Radiotherapy, radiofrequency ablation, and chemoembolization are of special value for patients with advanced adrenocortical carcinoma who cannot undergo an operation. Researchers have gradually realized the relationship between molecular changes in signalling pathways such as IGF2, Wnt/ $\beta$-catenin, and TP53-rb and the occurrence, development, recurrence, metastasis, and therapeutic effect of adrenocortical carcinoma[9]. Some molecular targeted drugs have been developed. Some researchers applied a humanized mouse model of adrenocortical carcinoma to explore the effect of anti-PD1 immunotherapy on the tumor microenvironment[10]. The results showed that the experimental group showed obvious tumor growth inhibition, and the tumor did not easily metastasize.

\section{Conclusions}

The incidence rate of adrenocortical oncocytic carcinoma is extremely low. CT scans and other imaging examination methods are not specific. For large adrenal tumors, the diagnosis of this disease should be considered. Surgical treatment should be the first choice after diagnosis. It is necessary to have regular follow-ups after the operation. 


\section{Abbreviations}

CT means Computed Tomography,Fig means Figure, MRI means Magnetic Resonance Imaging, NSE means Neuron specific enolase, Syn means Synaptophysin, Alk means Anaplastic Lymphoma Kinase, FDG means Fluorodeoxyglucose, PET means positron emission tomography, GLUT1 means Glucose Transporter 1, HPF means High power field, IGF means Insulin-like Growth Factor, TP means tumor protein, PD1 means programmed cell death protein 1

\section{Declarations}

Ethics approval and consent to participate: Not applicable.

Consent for publication: Written informed consent was obtained from the patient. The copyright to the English-language article is transferred to the journal of BMC Surgery when the article is accepted for publication.

Availability of data and materials: All data generated or analysed during this study are included in this published article.

Competing Interests: We have no competing interests.

Funding: This study was not supported by any financial funds. This study was not based on a previous communication at a society or meeting.

Authors Contributions: Liu Hongtao help date collection and pathological pictures. Huang Kai designed this study, wrote this manuscript and performed the operation. Wang Yehua is one surgeon involved in the operation. Gu Xiao help with manuscript revising.

Acknowledgements: We thank everyone who helped us with this manuscript.

\section{References}

1. Allolio B, Fassnacht M. Clinical review: Adrenocortical carcinoma: clinical update. J Clin Endocrinol Metab United States. 2006;91:2027-37.

2. Duregon E, Cappellesso R, Maffeis V, Zaggia B, Ventura L, Berruti A, et al. Validation of the prognostic role of the "Helsinki Score" in 225 cases of adrenocortical carcinoma. Hum Pathol United States. 2017;62:1-7.

3. Mills JK, Khalil M, Pasieka J, Kong S, Xu Y, Harvey A. Oncocytic subtypes of adrenal cortical carcinoma: Aggressive in appearance yet more indolent in behavior? Surgery. United States. 2019;166:524-33.

4. Libé R, Jazeron J-F, Louiset E, Groussin L. 18F-FDG PET reveals an adrenocortical carcinoma in a bilateral adrenal multinodular disease. Endocrine United States. 2019;63:188-9. 
5. Bisceglia M, Ludovico O, Di Mattia A, Ben-Dor D, Sandbank J, Pasquinelli G, et al. Adrenocortical oncocytic tumors: report of 10 cases and review of the literature. Int J Surg Pathol United States. 2004;12:231-43.

6. Renaudin K, Smati S, Wargny M, Al Ghuzlan A, Aubert S, Leteurtre E, et al. Clinicopathological description of 43 oncocytic adrenocortical tumors: importance of Ki-67 in histoprognostic evaluation. Mod Pathol an Off J United States Can Acad Pathol Inc. United States; 2018;31:1708-16.

7. Zlatibor L, Paunovic I, Zivaljevic V, Dundjerovic D, Tatic S, Djukic V. Prognostic significance of immunohistochemical markers in adrenocortical carcinoma. Acta Chir Belg England. 2020;120:23-9.

8. Paragliola RM, Torino F, Papi G, Locantore P, Pontecorvi A, Corsello SM. Role of Mitotane in Adrenocortical Carcinoma - Review and State of the art. Eur Endocrinol. 2018;14:62-6.

9. Crona J, Beuschlein F. Adrenocortical carcinoma - towards genomics guided clinical care. Nat Rev Endocrinol England. 2019;15:548-60.

10. Lang J, Capasso A, Jordan KR, French JD, Kar A, Bagby SM, et al. Development of an Adrenocortical Cancer Humanized Mouse Model to Characterize Anti-PD1 Effects on Tumor Microenvironment. J Clin Endocrinol Metab. United States; 2020;105.

\section{Figures}
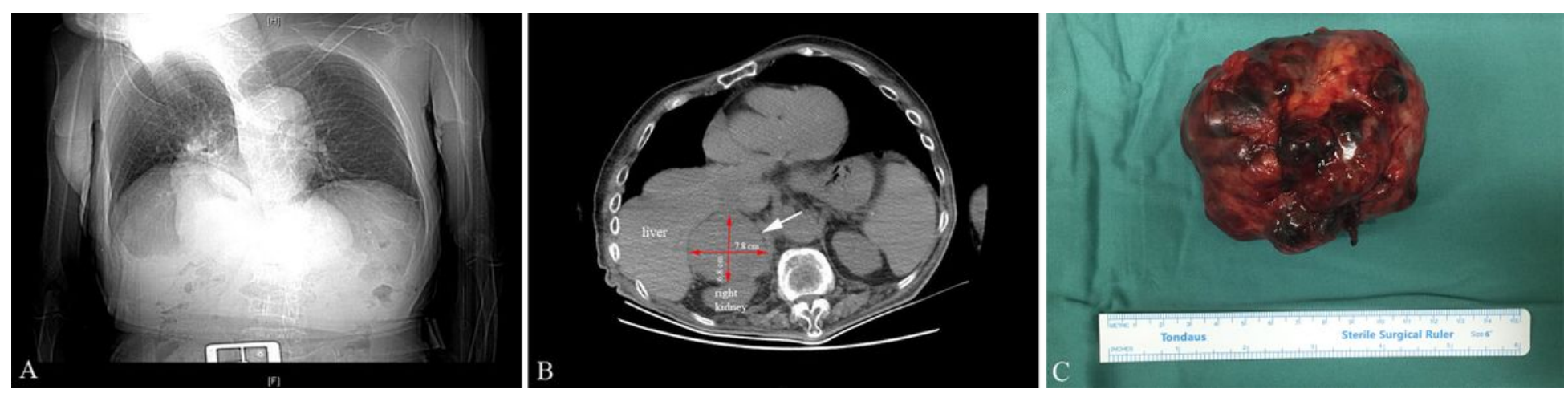

\section{Figure 1}

X-ray of chest shows that the patient had scoliosis $(A)$. The white arrow points to the location of the tumor. The red arrows indicate the length and width of the mass (B). Postoperative mass specimen of adrenocortical oncocytic carcinoma (C). 

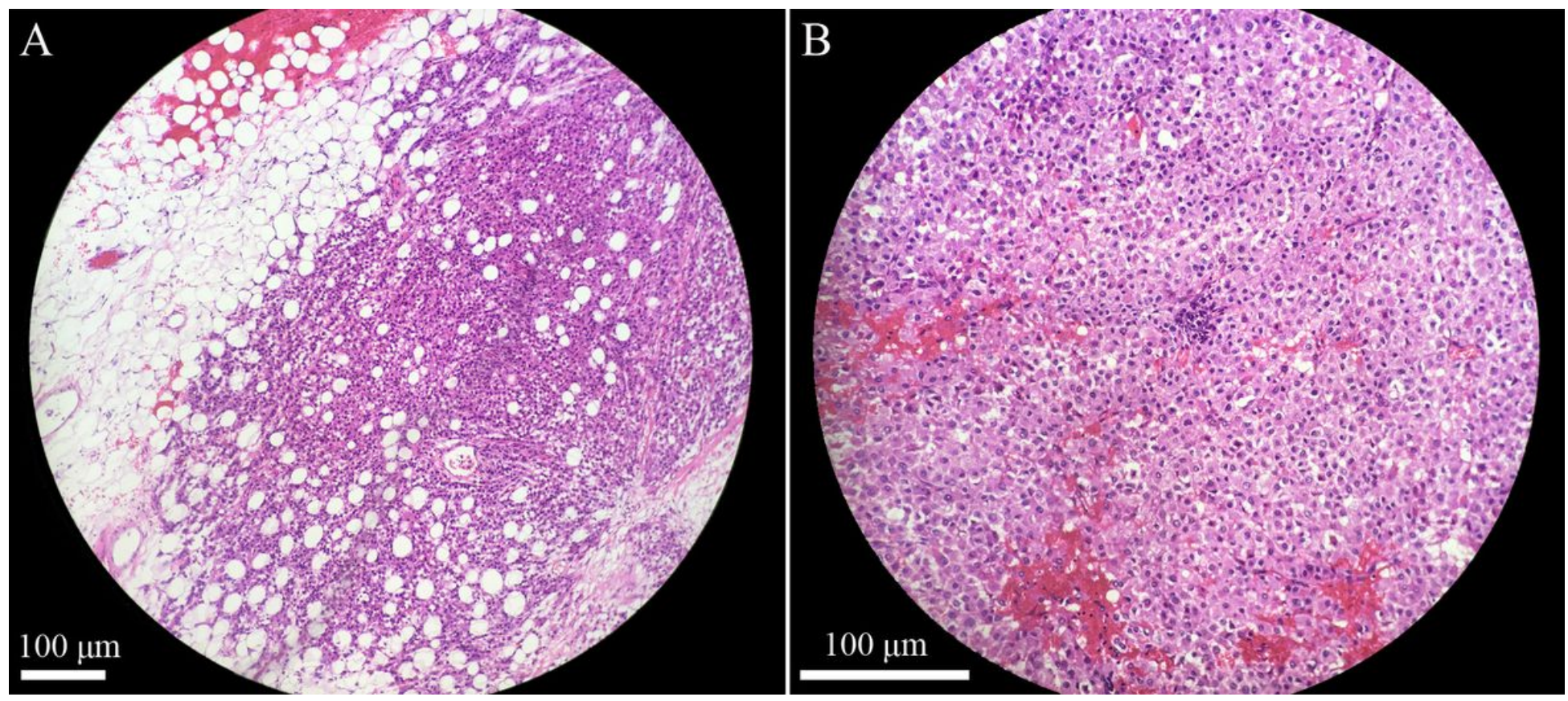

Figure 2

The tumor cells invaded adipose tissue, original amplification $\times 100(A)$. There were obvious stromal vessels with necrosis in tumor tissue, original amplification $\times 200(B)$

\section{Supplementary Files}

This is a list of supplementary files associated with this preprint. Click to download.

- renamed426cc.pdf 